\title{
A simple proof of the linear instability of rotating liquid drops
}

\author{
Mariarosaria Padula • Vsevolod A. Solonnikov
}

Received: 18 December 2007 / Accepted: 12 February 2008 / Published online: 25 April 2008

(C) The Author(s) 2008

\begin{abstract}
We give a simplified proof of the linear instability of equilibrium figures of rotating liquid based on energy estimates.
\end{abstract}

Keywords Nonlinear stability $\cdot$ Linear instability $\cdot$ Rotating drops $\cdot$ Self-gravitating incompressible fluids

Mathematics Subject Classification (2000) $\quad 76 \mathrm{D} \cdot 35 \mathrm{Q} \cdot 76 \mathrm{E}$

\section{Introduction}

The classical problem of stability of equilibrium figures of uniformly rotating viscous incompressible capillary self-gravitating liquid (see for instance [2]) is closely related to the free boundary problem governing the evolution of an isolated liquid mass. It consists of determination of a bounded domain $\Omega_{t} \in R^{3}$, the velocity vector field $\mathbf{V}(x, t)=\left(V_{1}, V_{2}, V_{3}\right)$, and the scalar pressure $q(x, t), x \in \Omega_{t}$, satisfying the following relations:

$$
\begin{aligned}
& \mathbf{V}_{t}+(\mathbf{V} \cdot \nabla) \mathbf{V}-v \nabla^{2} \mathbf{V}+\nabla q=\kappa \nabla U, \\
& \nabla \cdot \mathbf{V}=0, \quad x \in \Omega_{t}, \quad t>0, \\
& T(\mathbf{V}, q) \mathbf{n}=\sigma H(x, t) \mathbf{n}, \quad R_{n}=\mathbf{V} \cdot \mathbf{n}, \quad x \in \Gamma_{t} \equiv \partial \Omega_{t}, \\
& \mathbf{V}(x, 0)=\mathbf{V}_{0}, \quad x \in \Omega_{0} .
\end{aligned}
$$

M. Padula ( $\square)$

Dipartimento di Matematica, Università di Ferrara, via Machiavelli, 35, 44100 Ferrara, Italy e-mail:pad@unife.it

\section{A. Solonnikov}

Steklov Institute, via Fontanka 27, St. Petersburg, Russia

e-mail: solonnik@pdmi.ras.ru 
Here $v=$ const $>0$ is the viscosity coefficient, $\sigma$ and $\kappa$ are a non-negative coefficient of the surface tension and the gravitational constant, respectively, which do not vanish simultaneously, $T(\mathbf{V}, q)=-q I+v S(\mathbf{V})$ is the stress tensor, $S(\mathbf{V})=\left(\frac{\partial V_{j}}{\partial x_{k}}+\frac{\partial V_{k}}{\partial x_{j}}\right)$, $j, k=1,2,3$ is the doubled rate-of-strain tensor, $H$ is the doubled mean curvature of $\Gamma_{t}$ negative for convex domains, $R_{n}$ is the velocity of evolution of $\Gamma_{t}$ in the direction of the exterior normal $\mathbf{n}$ and

$$
U(x, t)=\int_{\Omega_{t}} \frac{d z}{|x-z|}
$$

is the Newtonian potential dependent on an unknown domain $\Omega_{t}$. The density of the liquid is assumed to be equal to one. The domain $\Omega_{0}$ is given.

Introducing a new pressure $q-\kappa U$ instead of $q$, that we shall continue to denote by $q$, we can write the above problem in the form

$$
\begin{gathered}
\mathbf{V}_{t}+(\mathbf{V} \cdot \nabla) \mathbf{V}-v \nabla^{2} \mathbf{V}+\nabla q=0, \\
\nabla \cdot \mathbf{V}=0, \quad x \in \Omega_{t}, t>0, \\
T(\mathbf{V}, q) \mathbf{n}=(\sigma H(x, t)+\kappa U(x, t)) \mathbf{n}, \quad R_{n}=\mathbf{V} \cdot \mathbf{n}, \quad x \in \Gamma_{t} \equiv \partial \Omega_{t}, \\
\mathbf{V}(x, 0)=\mathbf{V}_{0}, \quad x \in \Omega_{0} .
\end{gathered}
$$

From now on we shall put a prime to vectors $\mathbf{c} \equiv\left(c_{1}, c_{2}, c_{3}\right)$ to denote the part of $\mathbf{c}$ orthogonal to the unit vector of the $x_{3}$ axis $\mathbf{e}_{3}$, say $\mathbf{c}^{\prime} \equiv\left(c_{1}, c_{2}, 0\right)$. Let the liquid be rotating as a rigid body around a fixed axis ( $x_{3}$-axis), then the basic velocity vector field $\mathbf{V}_{b}$ and the pressure $P$ are given by

$$
\mathbf{V}_{b}(x)=\omega \mathbf{e}_{3} \times \mathbf{x}=\omega\left(-x_{2}, x_{1}, 0\right), \quad P(x)=\frac{\omega^{2}}{2}\left|x^{\prime}\right|^{2}+p_{0},
$$

where $x^{\prime}=\left(x_{1}, x_{2}, 0\right), p_{0}=$ const, and $\omega$ is the angular velocity of rotation. The domain $\mathscr{F}$ occupied by the rotating liquid, which is referred to as the equilibrium figure, is defined by the equation

$$
\sigma \mathscr{H}+\frac{\omega^{2}}{2}\left|x^{\prime}\right|^{2}+\kappa \mathscr{U}+p_{0}=0, \quad x \in \mathscr{G}=\partial \mathscr{F}
$$

where $\mathscr{H}$ is twice the mean curvature of $\mathscr{G}$, and

$$
\mathscr{U}(x)=\int_{\mathscr{F}} \frac{d z}{|x-z|} .
$$

The functions (1.2) satisfy the Navier-Stokes equations (1.1), and (1.3) is obtained by inserting (1.2), in place of $\mathbf{V}, q$, into the boundary condition $(1.1)_{3}$. If $\mathscr{F}$ is axially symmetric with respect to the $x_{3}$-axis, then $\mathbf{V}_{b}(x), P(x), x \in \mathscr{F}$, represent a stationary solution of the problem (1.1), otherwise these functions defined in a uniformly rotating 
domain $\mathscr{F}_{\omega t+\varphi}$ represent a periodic solution (by $\mathscr{F}_{\theta}$ we mean the domain obtained by rotation of the angle $\theta$ of $\mathscr{F}$ around the $x_{3}$-axis). Thus, the problem of stability of an equilibrium figure is equivalent to the problem of stability of a special stationary or periodic solution of (1.1).

We recall that solutions to (1.3) provide the stationary points of the functional

$$
E_{\beta}=\sigma|\Gamma|+\frac{\beta^{2}}{2 \int_{\Omega}\left|x^{\prime}\right|^{2} d x}-\frac{\kappa}{2} \int_{\Omega} \int_{\Omega} \frac{d x d y}{|x-y|}-p_{0}|\Omega|,
$$

where $\beta=\omega I_{3}$ is the magnitude of the total angular momentum of the rotating liquid and $I_{3}=\int_{\mathscr{F}}\left|\mathbf{e}_{3} \times x\right|^{2} d x$ is the inertia momentum of $\mathscr{F}$ with respect to the $x_{3}$-axis. The functional $E_{\beta}$ is considered in the set of domains $\Omega$, close to $\mathscr{F}$, with the same volume and the position of the barycenter as $\mathscr{F} ; \Gamma=\partial \Omega$. Equation (1.3) is the EulerLagrange equation associated with $E_{\beta}$. The second variation of $E_{\beta}$ is the quadratic form given by the formula (1.12) below. The equilibrium figure is regarded as stable if the second variation is positive and unstable if it can take negative values.

Justification of this statements is given in [6,8-12]. It is based on the analysis of the free boundary problem for the perturbations $\mathbf{W}=\left(\widehat{\mathbf{V}}-\widehat{\mathbf{V}}_{b}\right), s=q-P$, written in the coordinate system rotating with the angular velocity $\omega$ (see [9]):

$$
\begin{gathered}
\mathbf{W}_{t}+(\mathbf{W} \cdot \nabla) \mathbf{W}+2 \omega\left(e_{3} \times \mathbf{W}\right)-v \nabla^{2} \mathbf{W}+\nabla s=0, \\
\nabla \cdot \mathbf{W}=0, \quad y \in \widehat{\Omega}_{t}, \quad t>0, \\
T(\mathbf{W}, s) \widehat{\mathbf{n}}=\left(\sigma H(y)+\frac{\omega^{2}}{2}\left|y^{\prime}\right|^{2}+p_{0}+\kappa \widehat{U}(y, t)\right) \widehat{\mathbf{n}}, \\
\widehat{R_{n}}=\mathbf{W} \cdot \widehat{\mathbf{n}}, \quad y \in \widehat{\Gamma}_{t}, \\
\mathbf{W}(y, 0)=\widehat{\mathbf{V}}_{0}(y), \quad y \in \widehat{\Omega}_{0},
\end{gathered}
$$

where $y=\mathscr{Z}^{-1}\left(\Omega_{t}\right) x, \widehat{\Omega}_{t}=\mathscr{Z}^{-1}\left(\Omega_{t}\right) \Omega_{t}, \widehat{\Gamma}_{t}=\widehat{\partial \Omega}_{t}, \widehat{\mathbf{n}}$ is the exterior normal to $\widehat{\Gamma_{t}}, \widehat{U}(y, t)=\int_{\widehat{\Omega}_{t}}|y-z|^{-1} d z$ and

$$
\mathscr{Z}(\theta)=\left(\begin{array}{ccc}
\cos \theta & -\sin \theta & 0 \\
\sin \theta & \cos \theta & 0 \\
0 & 0 & 1
\end{array}\right)
$$

To the solution (1.2) of (1.1) corresponds the zero solution of (1.4).

In the present paper we are interested in the case of instability of this solution. We study the following evolution problem obtained by linearizing (1.4) around the given basic state $\mathbf{W}_{b}=0, \widehat{\Omega}_{t}=\mathscr{F}, \widehat{\Gamma}_{t}=\mathscr{G}$ :

$$
\begin{gathered}
\mathbf{v}_{t}+2 \omega\left(\mathbf{e}_{3} \times \mathbf{v}\right)-v \nabla^{2} \mathbf{v}+\nabla p=0, \\
\nabla \cdot \mathbf{v}(x, t)=0, \quad x \in \mathscr{F}, \quad t>0, \\
S(\mathbf{v})(x) \mathbf{N}(x)-\mathbf{N}(x)(\mathbf{N} \cdot S(\mathbf{v}) \mathbf{N})=0, \\
-p+v \mathbf{N} \cdot S(\mathbf{v}) \mathbf{N}+B_{0} \rho=0, \\
\rho_{t}=\mathbf{N}(x) \cdot \mathbf{v}(x, t)-h_{0}(x) \int_{\mathscr{G}} h_{0} \mathbf{N}(y) \cdot \mathbf{v}(y, t) d S, \quad x \in \mathscr{G}, \\
\mathbf{v}(x, 0)=\mathbf{v}_{0}(x), \quad x \in \mathscr{F}, \quad \rho(x, 0)=\rho_{0}(x), \quad x \in \mathscr{G} .
\end{gathered}
$$


Here $\mathbf{v}(x, t)=\left(v_{1}, v_{2}, v_{3}\right), p(x, t), x \in \mathscr{F}$, and $\rho(x, t), x \in \mathscr{G}=\partial \mathscr{F}$ are unknown functions, $\mathbf{N}$ is the exterior normal to $\mathscr{G}$, and

$$
h_{0}(x)=\frac{\mathbf{N} \cdot\left(\mathbf{e}_{3} \times x\right)}{\left\|\mathbf{N} \cdot\left(\mathbf{e}_{3} \times x\right)\right\|_{L_{2}(\mathscr{G})}} .
$$

If $\mathbf{N}(x) \cdot\left(\mathbf{e}_{3} \times x\right)=0$, which is the case for the axially symmetric $\mathscr{F}$, then $h_{0}=0$. Finally,

$$
B_{0} \rho(x, t)=-\sigma \Delta \mathscr{G} \rho(x, t)+b(x) \rho(x, t)-\kappa \int_{\mathscr{G}} \frac{\rho(y, t) d S}{|x-y|},
$$

where $\Delta_{\mathscr{G}}$ is the Laplace-Beltrami operator on $\mathscr{G}$,

$$
b(x)=\sigma\left(2 \mathscr{K}(x)-\mathscr{H}^{2}(x)\right)-\frac{\omega^{2}}{2} \frac{\partial}{\partial N}\left|x^{\prime}\right|^{2}-\kappa \frac{\partial}{\partial N} \int_{\mathscr{F}} \frac{d z}{|x-z|},
$$

$\mathscr{H}$ and $\mathscr{K}$ are the doubled mean curvature and the Gaussian curvature of $\mathscr{G}$, respectively. In the case $\sigma=0$, we assume

$$
b(x) \geq b_{0}>0 .
$$

The problem (1.5) is considered in the space of functions satisfying the following orthogonality conditions:

$$
\begin{aligned}
& \int_{\mathscr{G}} \rho(y, t) d S=0, \quad \int_{\mathscr{G}} \rho(y, t) y_{i} d S=0, \\
& \int_{\mathscr{F}} \mathbf{v}(y, t) d y=0, \\
& \int_{\mathscr{F}} \mathbf{v}(y, t) \cdot \eta_{i}(y) d y+\omega \int_{\mathscr{G}} \rho(y, t) \eta_{3}(y) \cdot \eta_{i}(y) d S=0, \quad i=1,2,3,
\end{aligned}
$$

where $\eta_{i}(x)=\mathbf{e}_{i} \times x$ and $\mathbf{e}_{i}$ is the unit vector directed along the $x_{i}$-axis. It is easily verified that the conditions (1.6) and (1.7) hold for arbitrary $t>0$ if they are satisfied at the initial moment $t=0$ for $\mathbf{v}=\mathbf{v}_{0}, \rho=\rho_{0}$. It is also obvious that $\int_{\mathscr{G}} \rho_{0} h_{0} d S=0$ implies

$$
\int_{\mathscr{G}} \rho(y, t) h_{0}(y) d S=0 .
$$


We assume that $\mathscr{F}$ is a given bounded domain in $\mathbb{R}^{3}$ with smooth boundary $\mathscr{G}$. It satisfies the conditions

$$
\int_{\mathscr{F}} x_{i} d x=0, \quad \int_{\mathscr{F}} x_{3} x_{j} d x=0, \quad i=1,2,3, \quad j=1,2
$$

(see [9]). Moreover, it may be assumed without loss of generality that

$$
\int_{\mathscr{F}} x_{1} x_{2} d x=0
$$

This relation is obvious if $\mathscr{F}$ is axially symmetric with respect to the $x_{3}$-axis; otherwise it can be achieved by rotation of $\mathscr{F}$ through an appropriate angle. Finally, we assume that

$$
\min _{|\theta| \leq \pi} \int_{\mathscr{F}}\left(\left(\cos \theta x_{1}+\sin \theta x_{2}\right)^{2}-x_{3}^{2}\right) d x>0,
$$

which means that $\mathscr{F}$ is an oblate domain. We prove the following theorem.

Theorem 1.1 Let

$$
\begin{gathered}
B \rho=B_{0} \rho+\frac{\omega^{2}\left|x^{\prime}\right|^{2}}{I_{3}} \int_{\mathscr{G}} \rho\left|y^{\prime}\right|^{2} d S, \\
\widehat{B} \rho=B \rho-\frac{1}{|\mathscr{G}|} \int_{\mathscr{G}} B \rho d S,
\end{gathered}
$$

and let conditions (1.9), (1.10) be satisfied. If the quadratic form

$$
\begin{aligned}
\int_{\mathscr{G}} \widehat{B} \rho \rho d S= & \int_{\mathscr{G}}\left(\sigma\left|\nabla_{\mathscr{G}} \rho\right|^{2}+b(x) \rho^{2}\right) d S \\
& -\kappa \int_{\mathscr{G}} \int_{\mathscr{G}} \frac{\rho(x) \rho(y) d S_{x} d S_{y}}{|x-y|}+\frac{\omega^{2}}{I_{3}}\left(\int_{\mathscr{G}} \rho(x)\left|x^{\prime}\right|^{2} d S\right)^{2},
\end{aligned}
$$

where $\nabla_{\mathscr{G}}$ is the surface gradient on $\mathscr{G}$, takes negative values for some $\rho(y)$ satisfying (1.6), (1.8), then the problem (1.5)-(1.8) has solutions growing exponentially as $t \rightarrow \infty$.

This means that the corresponding spectral problem has eigenvalues with positive real parts; this information is important for the proof of the instability of rigidly rotating liquid. In mechanics results of this type are referred to as "the inversion of the Lagrange theorem". 
The proof consists of the construction and estimate from below of a certain functional dependent on the solutions of (1.4) and playing the role of the Lyapunov function. It is much simpler than the proof given in [10-12]. The method we follow is proposed in [3-5] and used in [7] under the additional restriction $K$ er $\widetilde{B}=\emptyset$. Here this restriction is removed. Our approach to the proof of instability is similar to that in the papers [13] and [1], where other problems are studied and other Lyapunov functions are estimated.

\section{Auxiliary relations}

Before we proceed to the proof of the theorem, we introduce the following spaces of functions.

- $H$ : the subspace of functions $\rho \in L_{2}(\mathscr{G})$ satisfying (1.6);

- $\operatorname{Ker} \widehat{B}$ : the finite dimensional space of functions $\rho \in H$ satisfying $\widehat{B} \rho=0$ (in the case $\left.\sigma>0, \operatorname{Ker} \widehat{B} \subset W_{2}^{2}(\mathscr{G})\right)$.

- $H_{0}=H \ominus \operatorname{Ker} \widehat{B}$

- $H_{1}$ : the subspace of functions $\rho \in H_{0}$ satisfying the additional orthogonality conditions

$$
\int_{\mathscr{G}} \rho \eta_{j} \cdot \mathbf{N} d S=0, \quad j=1,2
$$

- $P_{0}, P_{1}$ : orthogonal projections on $H_{0}$ and $H_{1}$;

- $\quad(f, g)$ : the scalar product in $L_{2}(\mathscr{G})$.

Now, we present some important auxiliary relations the proof of which can be found in [12].

1. An arbitrary vector field of rigid motion $\eta(x)=\mathbf{a}+\mathbf{b} \times x, \mathbf{a}, \mathbf{b}=$ const, satisfies

$$
B \eta \cdot \mathbf{N}=-\omega^{2} \eta \cdot x^{\prime} .
$$

It follows that

$$
\int_{\mathscr{G}} \varphi(x) x_{1} x_{2} d S=\int_{\mathscr{G}} \varphi(x) x_{2} x_{3} d S=0, \quad \forall \varphi \in \operatorname{Ker} \widehat{B} .
$$

2. Let

$$
\tilde{\mathscr{S}}_{\alpha}=\int_{\mathscr{F}}\left(x_{\alpha}^{2}-x_{3}^{2}\right) d S, \quad \alpha=1,2
$$

By (1.10), $\widetilde{\mathscr{S}}_{\alpha}>0$. Direct computations show that

$$
\int_{\mathscr{G}} \eta_{1}(x) \cdot \mathbf{N}(x) x_{2} x_{3} d S=\tilde{\mathscr{S}}_{2}, \quad \int_{\mathscr{G}} \eta_{2}(x) \cdot \mathbf{N}(x) x_{1} x_{3} d S=-\tilde{\mathscr{S}}_{1},
$$




$$
\int_{\mathscr{G}} \eta_{1}(x) \cdot \mathbf{N}(x) x_{1} x_{3} d S=\int_{\mathscr{G}} \eta_{2}(x) \cdot \mathbf{N}(x) x_{2} x_{3} d S=0 .
$$

3. An arbitrary $\rho \in L_{2}(\mathscr{G})$ can be represented in the form

$$
\rho(x)=\rho_{1}(x)+\rho_{2}(x),
$$

where

$$
\begin{aligned}
& \rho_{1}(x)=\tilde{\mathscr{S}}_{2}^{-1} \eta_{1}(x) \cdot \mathbf{N}(x) I_{2}[\rho]-\tilde{\mathscr{S}}_{1}^{-1} \eta_{2}(x) \cdot \mathbf{N}(x) I_{1}[\rho], \\
& I_{\alpha}[\rho]=\int_{\mathscr{G}} \rho(x) x_{3} x_{\alpha} d S,
\end{aligned}
$$

and $\rho_{2}$ satisfies the orthogonality conditions

$$
\int_{\mathscr{G}} \rho_{2}(y) y_{3} y_{1} d S=\int_{\mathscr{G}} \rho_{2}(y) y_{3} y_{2} d S=0 .
$$

If $\rho \in H$, then $\rho_{2} \in H$. If $\rho=\eta_{\beta} \cdot \mathbf{N}, \beta=1,2$, then $\rho_{2}=0$.

4. By (1.9), (2.1) and (2.2), we have

$$
(\widehat{B} \rho, \rho)=\left(\widehat{B} \rho_{1}, \rho_{1}\right)+\left(\widehat{B} \rho_{2}, \rho_{2}\right),
$$

where

$$
\left(\widehat{B} \rho_{1}, \rho_{1}\right)=\left(B \rho_{1}, \rho_{1}\right)=\omega^{2} \sum_{\alpha=1}^{2} I_{\alpha}^{2}[\rho] \tilde{\mathscr{S}}_{\alpha}^{-1} .
$$

By (1.10), this quadratic form is positive, whence

$$
(\widehat{B} \rho, \rho) \geq\left(\widehat{B} \rho_{2}, \rho_{2}\right) .
$$

5. The equation (2.3) defines a non-orthogonal projection $Q$ on the space of functions $\rho$ satisfying (2.4):

$$
Q \rho=\rho-\left(\tilde{\mathscr{S}}_{2}^{-1} \eta_{1}(x) \cdot \mathbf{N}(x) I_{2}[\rho]-\tilde{\mathscr{S}}_{1}^{-1} \eta_{2}(x) \cdot \mathbf{N}(x) I_{1}[\rho]\right)=\rho_{2} .
$$

The adjoint projection has the form

$$
Q^{*} \rho=\rho+\left(\tilde{\mathscr{S}}_{1}^{-1} x_{1} x_{3} J_{2}[\rho]-\tilde{\mathscr{S}}_{2}^{-1} x_{2} x_{3} J_{1}[\rho]\right),
$$

where

$$
J_{\alpha}[\rho]=\int_{\mathscr{G}} \eta_{\alpha} \cdot \mathbf{N} \rho d S, \quad \alpha=1,2 .
$$


It is a projection on the space defined by

$$
J_{\alpha}[\rho]=0, \quad \alpha=1,2 .
$$

6. If $\rho \in H_{0}$, then

$$
\rho=P_{0} \rho=P_{0} \rho_{1}+P_{0} Q \rho,
$$

and $P_{0} Q \rho$ is representable in a unique way in the form

$$
P_{0} Q \rho=P_{0} Q r
$$

where

$$
r=P_{1} Q \rho \in H_{1} \text {. }
$$

7. By (2.6), for $\rho \in H_{0}$

$$
(\widehat{B} \rho, \rho) \geq\left(\widehat{B} P_{0} Q \rho, P_{0} Q \rho\right)=(\widehat{B} Q r, Q r) .
$$

Thus, if the form (1.12) can take negative values, then the same is true for the form $(\widehat{B} Q r, Q r)$, which means that the operator $B_{1}=Q^{*} \widehat{B} Q$ restricted to $H_{1}$, i.e. the operator $\widetilde{B}=P_{1} Q^{*} \widehat{B} Q P_{1}$, has negative eigenvalues. Only a finite number of them may exist. Moreover,

$$
\operatorname{Ker} \widetilde{B}=\emptyset
$$

hence

$$
H_{1}=H_{+} \oplus H_{-},
$$

where $H_{-}$is the finite-dimensional space spanned by eigenfunctions of $\widetilde{B}$ corresponding to the negative eigenvalues. We have

$$
(\widehat{B} Q r, Q r)=\left(\widehat{B} Q r_{+}, Q r_{+}\right)+\left(\widehat{B} Q r_{-}, Q r_{-}\right),
$$

where $r_{ \pm}=P_{ \pm} r$ and $P_{ \pm}$are orthogonal projections on $H_{ \pm}$. For $r_{ \pm} \neq 0$, the first-term on the right-hand side is positive and the second-term is negative. For an arbitrary $s \in L_{2}(\mathscr{G})$, the equations

$$
B_{1} r_{ \pm}=P_{ \pm} S
$$

are uniquely solvable in $H_{1}$ and $r_{ \pm} \in H_{ \pm}$.

Finally, we use the following proposition (for instance, see [8]): 
Proposition 2.1 For an arbitrary $f \in W_{2}^{1 / 2}(\mathscr{G}) \cap H$ there exists a solenoidal vector field $\mathbf{w} \in W_{2}^{1}(\mathscr{F})$ such that $\left.\mathbf{w} \cdot \mathbf{N}\right|_{\mathscr{G}}=f$,

$$
\int_{\mathscr{F}} \mathbf{w} \cdot \eta_{i} d x=0, \quad \eta_{i}=\mathbf{e}_{i} \times x, \quad i=1,2,3,
$$

and

$$
\begin{aligned}
& \|\mathbf{w}\|_{W_{2}^{1}(\mathscr{F})} \leq c\|f\|_{W_{2}^{1 / 2}(\mathscr{G})}, \\
& \|\mathbf{w}\|_{L_{2}(\mathscr{F})} \leq c\|f\|_{L_{2}(\mathscr{G})} .
\end{aligned}
$$

The correspondence between $f$ and $\mathbf{w}$ is linear.

\section{Proof of Theorem 1.1}

Now, we pass to the proof of Theorem 1.1. We transform the problem (1.5)-(1.8). In view of (1.7), we have

$$
\mathbf{v}(x, t)=\mathbf{v}^{\perp}(x, t)+\sum_{i=1}^{3} d_{i}[\rho] \eta_{i}(x)
$$

where $\mathbf{v}^{\perp}$ is a vector field orthogonal to any rigid motion $\eta=\mathbf{a}+\mathbf{b} \times x$ defined at point 1 . of Section 2 , with $\eta_{i}$ defined after formula (1.7). Namely $\mathbf{v}^{\perp}$ satisfies:

$$
\int_{\mathscr{F}} \mathbf{v}^{\perp} \cdot \eta(x) d x=0
$$

moreover

$$
d_{i}[\rho]=-\frac{\omega}{\left\|\eta_{i}\right\|_{L_{2}(\mathscr{F})}^{2}} \int_{\mathscr{G}} \rho(y, t) \eta_{i}(y) \cdot \eta_{3}(y) d S .
$$

We introduce the functions

$$
\mathbf{u}(x, t)=\mathbf{v}(x, t)-d_{3}[\rho] \eta_{3}(x), \quad q(x, t)=p(x, t)-\omega d_{3}[\rho]\left|x^{\prime}\right|^{2}+\text { const } .
$$

Since $2 \mathbf{e}_{3} \times \eta_{3}(x)=-2\left(x_{1}, x_{2}, 0\right)=-\nabla\left|x^{\prime}\right|^{2}$ and

$$
\frac{\partial}{\partial t} d_{3}[\rho]=-\frac{\omega}{\left\|\eta_{3}\right\|_{L_{2}(\mathscr{F})}^{2}} \int_{\mathscr{G}} \mathbf{v} \cdot \mathbf{N}\left|\eta_{3}(y)\right|^{2} d S=-\frac{2 \omega}{\left\|\eta_{3}\right\|_{L_{2}(\mathscr{F})}^{2}} \int_{\mathscr{F}} \mathbf{u} \cdot x^{\prime} d x
$$


relations (1.5) can be written in the form

$$
\begin{gathered}
\mathbf{u}_{t}+2 \omega\left(\mathbf{e}_{3} \times \mathbf{u}\right)-v \nabla^{2} \mathbf{u}+\nabla q=\frac{2 \omega \eta_{3}(x)}{\left\|\eta_{3}\right\|_{L_{2}(\mathscr{F})}^{2}} \int_{\mathscr{F}} \mathbf{u} \cdot x^{\prime} d x, \\
\nabla \cdot \mathbf{u}(x, t)=0, \quad x \in \mathscr{F}, \quad t>0, \\
S(\mathbf{u}(x)) \mathbf{N}(x)-\mathbf{N}(x)(\mathbf{N} \cdot S(\mathbf{u}) \mathbf{N})=0, \\
-q+v \mathbf{N} \cdot S(\mathbf{u}) \mathbf{N}+\widehat{B} \rho=0, \\
\rho_{t}=\mathbf{N}(x) \cdot \mathbf{u}(y, t)-h_{0}(x) \int_{\mathscr{G}} h_{0} \mathbf{N}(y) \cdot \mathbf{u}(y, t) d S, \quad x \in \mathscr{G}, \\
\mathbf{u}(x, 0)=\mathbf{v}_{0}(x)-\mathbf{u}(x, 0) \equiv \mathbf{u}_{0}(x), \quad x \in \mathscr{F}, \quad \rho(x, 0)=\rho_{0}(x), \quad x \in \mathscr{G} .
\end{gathered}
$$

Orthogonality conditions (1.6) remain invariant and (1.7) are converted to

$$
\begin{aligned}
& \int_{\mathscr{F}} \mathbf{u}(x, t) d x=0, \quad \int_{\mathscr{F}} \mathbf{u}(x, t) \cdot \eta_{3}(x) d x=0, \\
& \int_{\mathscr{F}} \mathbf{u}(x, t) \cdot \eta_{\alpha}(x) d x+\omega \int_{\mathscr{G}} \rho(x, t) \eta_{3}(x) \cdot \eta_{\alpha}(x) d S=0, \quad \alpha=1,2 .
\end{aligned}
$$

Now we change the equation for $\rho_{t}$ and instead of (3.1) consider the problem

$$
\begin{gathered}
\mathbf{u}_{t}+2 \omega\left(\mathbf{e}_{3} \times \mathbf{u}\right)-v \nabla^{2} \mathbf{u}+\nabla q=\frac{2 \omega \eta_{3}(x)}{\left\|\eta_{3}\right\|_{L_{2}(\mathscr{F})}^{2}} \int_{\mathscr{F}} \mathbf{u} \cdot x^{\prime} d x, \\
\nabla \cdot \mathbf{u}(x, t)=0, \quad x \in \mathscr{F}, \quad t>0, \\
S(\mathbf{u}) \mathbf{N}(x)-\mathbf{N}(x)(\mathbf{N} \cdot S(\mathbf{u}) \mathbf{N})=0, \\
-q+v \mathbf{N} \cdot S(\mathbf{u}) \mathbf{N}+\widehat{B} \rho=0, \\
\rho_{t}=P_{0} \mathbf{N}(x) \cdot \mathbf{u}(x, t), \quad x \in \mathscr{G}, \\
\mathbf{u}(x, 0)=\mathbf{u}_{0}(x), \quad x \in \mathscr{F}, \quad \rho(x, 0)=\rho_{0}(x)=P_{0} \rho_{0}, \quad x \in \mathscr{G} .
\end{gathered}
$$

We subject the initial data $\mathbf{u}_{0}, \rho_{0}$ to the conditions (3.2). It can be shown that in this case the same relations hold for all $t>0$. If $(\mathbf{u}, p, \rho)$ is a solution of (3.3) that grows exponentially as $t \rightarrow \infty$, then $\left(\mathbf{u}, r, \rho^{\prime}\right)$ with

$$
\rho^{\prime}=\rho+P^{\prime} \int_{0}^{t} \mathbf{u}(x, \tau) \cdot \mathbf{N}(x) d \tau
$$

is a growing solution of (1.5)-(1.8); by $P^{\prime}$ we mean the projection on the space $\operatorname{Ker} \widehat{B} \ominus h_{0}$ (we recall that $\left.h_{0} \in \operatorname{Ker} \widehat{B}\right)$. 
We follow the arguments in [7] and construct functions $E(t)$ and $E_{1}(t)$ such that

$$
\begin{aligned}
& \frac{d E(t)}{d t}+E_{1}(t)=0, \\
& E_{1}(t) \geq-\beta E(t), \quad E(0)<0, \quad \beta>0 .
\end{aligned}
$$

Then $z(t)=-E(t)$ satisfies $\frac{d z(t)}{d t}>\beta z(t)$ and, as a consequence,

$$
z(t) \geq z(0) e^{\beta t}
$$

which proves the theorem.

We obtain some estimates of $\mathbf{u}$ and $\rho$ that are uniform with respect to $t$. We multiply the first equation in (3.3) by $\mathbf{u}$ and integrate over $\mathscr{F}$. Then we integrate by parts and make use of the boundary conditions. This leads to

$$
\frac{1}{2} \frac{d}{d t}\|\mathbf{u}(\cdot, t)\|_{L_{2}(\mathscr{F})}^{2}+(\widehat{B} \rho, \mathbf{u} \cdot \mathbf{N})+\frac{v}{2}\|S(\mathbf{u})\|_{L_{2}(\mathscr{F})}^{2}=0
$$

Since

$$
(\widehat{B} \rho, \mathbf{u} \cdot \mathbf{N})=\left(\widehat{B} \rho, P_{0} \mathbf{u} \cdot \mathbf{N}\right)=\left(\widehat{B} \rho, \rho_{t}\right),
$$

we obtain the energy relation

$$
\frac{1}{2} \frac{d}{d t}\left(\|\mathbf{u}(\cdot, t)\|_{L_{2}(\mathscr{F})}^{2}+(\widehat{B} \rho, \rho)\right)+\frac{v}{2}\|S(\mathbf{u})\|_{L_{2}(\mathscr{F})}^{2}=0 .
$$

In view of the orthogonality conditions, we have

$$
\|\mathbf{u}\|_{L_{2}(\mathscr{F})}^{2}=\left\|\mathbf{v}^{\perp}\right\|_{L_{2}(\mathscr{F})}^{2}+\sum_{\alpha=1}^{2} d_{\alpha}^{2}\left\|\eta_{\alpha}\right\|_{L_{2}(\mathscr{F})}^{2}
$$

and, as a consequence,

$$
\begin{aligned}
& \|\mathbf{u}(\cdot, t)\|_{L_{2}(\mathscr{F})}^{2}+(\widehat{B} \rho, \rho) \\
& =\left\|\mathbf{v}^{\perp}(\cdot, t)\right\|_{L_{2}(\mathscr{F})}^{2}+\left(B \rho_{1}, \rho_{1}\right)+\sum_{\alpha=1}^{2} d_{\alpha}^{2}\left\|\eta_{\alpha}\right\|_{L_{2}(\mathscr{F})}^{2} \\
& \quad+\left(\widehat{B} Q r_{+}, Q r_{+}\right)+\left(\widehat{B} Q r_{-}, Q r_{-}\right),
\end{aligned}
$$

where $r=P_{1} Q \rho, r_{ \pm}=P_{ \pm} r$ and $\rho_{1}$ is the same as in (2.3).

Next, we use the relations

$$
2 \mathbf{e}_{3} \times \eta_{\alpha}=2 \mathbf{e}_{\alpha} x_{3}=\mathbf{e}_{\alpha} x_{3}-\mathbf{e}_{3} x_{\alpha}+\nabla x_{3} x_{\alpha}, \quad \alpha=1,2,
$$


and write the first equation in (3.3) in the form

$$
\mathbf{v}_{t}^{\perp}+2 \omega\left(\mathbf{e}_{3} \times \mathbf{v}^{\perp}\right)-v \nabla^{2} \mathbf{v}^{\perp}+\nabla\left(q+\omega \sum_{\alpha=1}^{2} d_{\alpha} x_{\alpha} x_{3}\right)=\mathbf{R},
$$

where $\mathbf{R}$ is a linear combination of $\eta_{i}$. We multiply (3.7) by the vector field $\mathbf{w}$, introduced in Proposition 2.1, with

$$
f=P_{0}\left(I_{2}[\rho] \eta_{1} \cdot \mathbf{N}-I_{1}[\rho] \eta_{2} \cdot \mathbf{N}+Q s\right), \quad s \in H_{1} \cap W_{2}^{1 / 2}(\mathscr{G}),
$$

and integrate over $\mathscr{F}$. Upon integration by parts we arrive at

$$
\begin{aligned}
& \frac{d}{d t} \int_{\mathscr{F}} \mathbf{v}^{\perp} \cdot \mathbf{w} d x-\int_{\mathscr{F}} \mathbf{v}^{\perp} \cdot \mathbf{w}_{t} d x+2 \omega \int_{\mathscr{F}}\left(\mathbf{e}_{3} \times \mathbf{v}^{\perp}\right) \cdot \mathbf{w} d x \\
& +\frac{v}{2} \int_{\mathscr{F}} S\left(\mathbf{v}^{\perp}\right): S(\mathbf{w}) d x+\int_{\mathscr{G}}\left(\widehat{B} \rho+\omega \sum_{\alpha=1}^{2} d_{\alpha}[\rho] x_{3} x_{\alpha}\right) f d S=0 .
\end{aligned}
$$

Equation (3.2) is obtained by adding (3.6) and (3.8) multiplied by a small $\gamma>0$. We have

$$
\begin{aligned}
E(t)= & \frac{1}{2}\left(\left\|\mathbf{v}^{\perp}(\cdot, t)\right\|_{L_{2}(\mathscr{F})}^{2}+\left(B \rho_{1}, \rho_{1}\right)+\sum_{\alpha=1}^{2} d_{\alpha}^{2}[\rho]\left\|\eta_{\alpha}\right\|_{L_{2}(\mathscr{F})}^{2}\right. \\
& \left.+\left(\widehat{B} Q r_{+}, Q r_{+}\right)+\left(\widehat{B} Q r_{-}, Q r_{-}\right)+2 \gamma \int_{\mathscr{F}} \mathbf{v}^{\perp} \cdot \mathbf{w} d x\right), \\
E_{1}(t)= & \frac{v}{2}\left\|S\left(\mathbf{v}^{\perp}\right)\right\|_{L_{2}(\mathscr{F})}^{2}-\gamma \int_{\mathscr{F}} \mathbf{v}^{\perp} \cdot \mathbf{w}_{t} d x+2 \omega \gamma \int_{\mathscr{F}}\left(\mathbf{e}_{3} \times \mathbf{v}^{\perp}\right) \cdot \mathbf{w} d x \\
& +\frac{v \gamma}{2} \int_{\mathscr{F}} S\left(\mathbf{v}^{\perp}\right): S(\mathbf{w}) d x+\gamma I_{\mathscr{G}},
\end{aligned}
$$

where $I_{\mathscr{G}}$ is the surface integral in (3.8).

Now we verify (3.5). We use the Korn inequality

$$
\left\|\mathbf{v}^{\perp}\right\|_{W_{2}^{1}(\mathscr{F})} \leq c\left\|S\left(\mathbf{v}^{\perp}\right)\right\|_{L_{2}(\mathscr{F})}
$$

and the estimates (2.7) for w. We distinguish two cases: $\sigma>0$ and $\sigma=0$.

I. $\sigma>0$. We set $s=r_{+}-r_{-}$. By (2.7),

$$
\|\mathbf{w}\|_{L_{2}(\mathscr{F})} \leq c\|f\|_{L_{2}(\mathscr{G})} \leq c\left(\sum_{\alpha=1}^{2} I_{\alpha}^{2}[\rho]+\left\|r_{+}\right\|_{L_{2}(\mathscr{G})}^{2}+\left\|r_{-}\right\|_{L_{2}(\mathscr{G})}^{2}\right)^{1 / 2} .
$$


Using the Cauchy-Schwartz inequality, we easily show that

$$
E(t) \geq c\left(\widehat{B} Q r_{-}, Q r_{-}\right)
$$

in the case of small $\gamma>0$.

We pass to the estimate of $E_{1}(t)$. Since $\rho(x, t)=P_{0} \rho(x, t)$ for all $t \geq 0$, we have

$$
B \rho+\omega \sum_{\alpha=1}^{2} d_{\alpha} x_{3} x_{\alpha}=B Q \rho+\omega^{2} \sum_{\alpha=1}^{2} M_{\alpha} \widetilde{S}_{\alpha}^{-1} I_{\alpha}[\rho] x_{3} x_{\alpha}
$$

with $M_{\alpha}=\left\|\eta_{3}\right\|_{L_{2}(\mathscr{F})}^{2}\left\|\eta_{\alpha}\right\|_{\left.L_{2} \mathscr{F}\right)}^{-2}$. It follows that the surface integral $I_{\mathscr{G}}$ can be written in the form

$$
\begin{aligned}
I_{\mathscr{G}} & =\omega^{2} \sum_{\alpha=1}^{2} M_{\alpha} I_{\alpha}^{2}[\rho]+(\widehat{B} Q r, Q s) \\
& =\omega^{2} \sum_{\alpha=1}^{2} M_{\alpha} I_{\alpha}^{2}[\rho]+\left(\widehat{B} Q r_{+}, Q r_{+}\right)-\left(\widehat{B} Q r_{-}, Q r_{-}\right)
\end{aligned}
$$

The sum of the last two terms is equivalent to $\|r\|_{W_{2}^{1}(\mathscr{G})}^{2}$, whence

$$
I_{\mathscr{G}} \geq c\left(\sum_{\alpha=1}^{2} I_{\alpha}^{2}[\rho]+\|r\|_{W_{2}^{1}(\mathscr{G})}^{2}\right)
$$

We also need to estimate $\mathbf{w}_{t}$. By (2.7),

$$
\left\|\mathbf{w}_{t}\right\|_{L_{2}(\mathscr{F})} \leq c\left\|f_{t}\right\|_{L_{2}(\mathscr{G})}
$$

The time derivative $f_{t}$ can be written in the form

$$
\begin{aligned}
f_{t} & =P_{0}\left(I_{2}\left[\rho_{t}\right] \eta_{1} \cdot \mathbf{N}-I_{1}\left[\rho_{t}\right] \eta_{2} \cdot \mathbf{N}\right)+P_{0} Q r_{t}-2 P_{0} Q r_{-, t} \\
& =P_{0}\left(I_{2}\left[\rho_{t}\right] \eta_{1} \cdot \mathbf{N}-I_{1}\left[\rho_{t}\right] \eta_{2} \cdot \mathbf{N}\right)+P_{1} Q \rho_{t}-2 P_{0} Q r_{-, t} .
\end{aligned}
$$

Since $P_{0} \eta_{3} \cdot \mathbf{N}=0$, we have

$$
\rho_{t}=P_{0}\left(\mathbf{v}^{\perp} \cdot \mathbf{N}+\sum_{\alpha=1}^{2} d_{\alpha}[\rho] \eta_{\alpha} \cdot \mathbf{N}\right) .
$$

As for the last term in (3.11), we use the formula

$$
r_{-, t}=\sum_{j=1}^{m}\left(r_{t}, \varphi_{j}\right) \varphi_{j}(x)=\sum_{j=1}^{m}\left(P_{1} Q \rho_{t}, \varphi_{j}\right) \varphi_{j}(x),
$$


where the $\varphi_{j}$ are normalized eigenfunctions of the operator $B_{1}$ corresponding to the negative eigenvalues. We can estimate a finite sum (3.13) using (3.12). We obtain

$$
\left\|\rho_{t}\right\|_{L_{2}(\mathscr{G})} \leq c\left(\sum_{\alpha=1}^{2} I_{\alpha}^{2}[\rho]+\left\|\mathbf{v}^{\perp} \cdot \mathbf{N}\right\|_{L_{2}(\mathscr{G})}^{2}\right)^{1 / 2}
$$

and

$$
\left\|\mathbf{w}_{t}\right\|_{L_{2}(\mathscr{F})} \leq c\left\|f_{t}\right\|_{L_{2}(\mathscr{G})} \leq c\left(\sum_{\alpha=1}^{2} I_{\alpha}^{2}[\rho]+\left\|S\left(\mathbf{v}^{\perp}\right)\right\|_{L_{2}(\mathscr{F})}^{2}\right)^{1 / 2}
$$

Finally, by (2.6),

$$
\|S(\mathbf{w})\|_{L_{2}(\mathscr{F})}^{2} \leq c\|f\|_{W_{2}^{1 / 2}(\mathscr{G})}^{2} \leq c\left(\sum_{\alpha=1}^{2} I_{\alpha}^{2}[\rho]+\|r\|_{W_{2}^{1 / 2}(\mathscr{G})}^{2}\right) .
$$

Estimates (3.9), (3.14) and (3.15) enable us to show, using the Cauchy-Schwartz inequality, that

$$
\begin{aligned}
& \left|-\gamma \int_{\mathscr{F}} \mathbf{v}^{\perp} \cdot \mathbf{w}_{t} d x+2 \omega \gamma \int_{\mathscr{F}}\left(\mathbf{e}_{3} \times \mathbf{v}^{\perp}\right) \cdot \mathbf{w} d x+\frac{\nu \gamma}{2} \int_{\mathscr{F}} S\left(\mathbf{v}^{\perp}\right): S(\mathbf{w}) d x\right| \\
& \quad \leq \theta\left(\frac{v}{2}\left\|S\left(\mathbf{v}^{\perp}\right)\right\|_{L_{2}(\mathscr{F})}^{2}+\gamma I_{\mathscr{G}}\right)
\end{aligned}
$$

with $\theta \in(0,1)$. It follows that

$$
E_{1}(t) \geq c\left\|r_{-}\right\|_{W_{2}^{1}(\mathscr{G})}^{2} \geq-\beta E(t)
$$

if $\gamma$ is sufficiently small. At the initial moment $t=0$ we have

$$
\begin{aligned}
E(0)= & \frac{1}{2}\left(\left\|\mathbf{v}^{\perp}(\cdot, 0)\right\|_{L_{2}(\mathscr{F})}^{2}+\left(B \rho_{1}, \rho_{1}\right)+\sum_{\alpha=1}^{2} d_{\alpha}^{2}[\rho]\left\|\eta_{\alpha}\right\|_{L_{2}(\mathscr{F})}^{2}\right. \\
& \left.+\left(\widehat{B} Q r_{+}, Q r_{+}\right)+\left(\widehat{B} Q r_{-}, Q r_{-}\right)+2 \gamma \int_{\mathscr{F}} \mathbf{v}^{\perp} \cdot \mathbf{w} d x\right)_{t=0} \\
= & \left.\left(\widehat{B} Q r_{-}, Q r_{-}\right)\right|_{t=0}<0
\end{aligned}
$$

if $\mathbf{v}^{\perp}(x, 0)=0$ and $\rho(x, 0)=P_{0} Q \rho(x, 0)=P_{0} Q r_{-}(x, 0)$. Hence in the case $\sigma>0$ (3.5) is proved. 
II. $\sigma=0$. We set $s=s_{+}+s_{-}$where $s_{ \pm} \in H_{ \pm}$are solutions of the equations

$$
B_{1} s_{ \pm}=P_{ \pm}(-\Delta \mathscr{G})^{-1 / 2} r_{ \pm}
$$

where $\Delta \mathscr{G}$ is the Laplace Beltrami operator. These functions satisfy the inequalities

$$
\left\|s_{ \pm}\right\|_{W_{2}^{1 / 2}(\mathscr{G})} \leq c\left\|(-\Delta \mathscr{G})^{-1 / 2} r_{ \pm}\right\|_{W_{2}^{1 / 2}(\mathscr{G})} \leq c\left\|r_{ \pm}\right\|_{W_{2}^{-1 / 2}(\mathscr{G})}
$$

so instead of (3.9) we have

$$
\|\mathbf{w}\|_{L_{2}(\mathscr{F})} \leq c\|f\|_{L_{2}(\mathscr{G})} \leq c\left(\sum_{\alpha=1}^{2} d_{\alpha}^{2}[\rho]+\left\|r_{+}\right\|_{W_{2}^{-1 / 2}(\mathscr{G})}^{2}+\left\|r_{-}\right\|_{W_{2}^{-1 / 2}(\mathscr{G})}^{2}\right)^{1 / 2} .
$$

Estimate (3.10) remains valid if $\gamma$ is small. Instead of (3.11) and (3.15) we have

$$
\begin{aligned}
& \|S(\mathbf{w})\|_{L_{2}(\mathscr{F})}^{2} \leq c\|f\|_{W_{2}^{1 / 2}(\mathscr{G})}^{2} \leq c\left(\sum_{\alpha=1}^{2} I_{\alpha}^{2}[\rho]+\|r\|_{W_{2}^{-1 / 2}(\mathscr{G})}^{2}\right), \\
& f_{t}=P_{0}\left(I_{2}\left[\rho_{t}\right] \eta_{1} \cdot \mathbf{N}-I_{1}\left[\rho_{t}\right] \eta_{2} \cdot \mathbf{N}\right)+P_{0} Q s_{t}-2 P_{0} Q s_{-, t}, \\
& \left\|f_{t}\right\|_{L_{2}(\mathscr{G})} \leq c\left(\sum_{\alpha=1}^{2} I_{\alpha}^{2}\left[\rho_{t}\right]+\left\|r_{t}\right\|_{W_{2}^{-1 / 2}(\mathscr{G})}^{2}+\left\|r_{-, t}\right\|_{W_{2}^{-1 / 2}(\mathscr{G})}^{2}\right)^{1 / 2} .
\end{aligned}
$$

We estimate the norms of $r_{t}$ and $r_{-, t}$, using (3.12) and (3.13), which leads to the same inequality (3.14) as in the case $\sigma>0$. The surface integral $I_{\mathscr{G}}$ has the form

$$
I_{\mathscr{G}}=\omega^{2} \sum_{\alpha=1}^{2} M_{\alpha} I_{\alpha}^{2}[\rho]+(\widehat{B} Q r, Q s),
$$

where

$$
(\widehat{B} Q r, Q s)=\left(r, Q^{*} \widehat{B} Q s\right)=\left(r_{+},(-\Delta \mathscr{G})^{-1 / 2} r_{+}\right)+\left(r_{-},(-\Delta \mathscr{G})^{-1 / 2} r_{-}\right) .
$$

Hence

$$
I_{\mathscr{G}} \geq c\left(\sum_{\alpha=1}^{2} I_{\alpha}^{2}[\rho]+\|r\|_{W_{2}^{-1 / 2}(\mathscr{G})}^{2}\right) .
$$

It follows that (3.16) holds also in the case $\sigma=0$, whence

$$
E_{1}(t) \geq c\left\|r_{-}\right\|_{W_{2}^{-1 / 2}(\mathscr{G})}^{2} .
$$


Finally, we use the fact that

$$
\left\|r_{-}\right\|_{L_{2}(\mathscr{G})}^{2}=\sum_{j=1}^{m}\left|\left(r, \varphi_{j}\right)\right|^{2} \leq\|r\|_{W_{2}^{-1 / 2}(\mathscr{G})}^{2} \sum_{j=1}^{m}\left\|\varphi_{j}\right\|_{W_{2}^{1 / 2}(\mathscr{G})}^{2}
$$

and $m<\infty$. This implies $E_{1}(t) \geq c\left\|r_{-}\right\|_{L_{2}(\mathscr{G})}^{2} \geq-c\left(\widehat{B} Q r_{-}, Q r_{-}\right) \geq-\beta E(t)$, which was required. Theorem 1.1 is completely proved.

Acknowledgments Padula thanks Italian MURST 60\%, 40\% Preziosi, "Modelli matematici di crescita e vascolarizzazioni di tumori e tessuti biologici" MURST, and GNFM of italian CNR-INDAM.

Open Access This article is distributed under the terms of the Creative Commons Attribution Noncommercial License which permits any noncommercial use, distribution, and reproduction in any medium, provided the original author(s) and source are credited.

\section{References}

1. Abeyaratne, R., Knowles, J.K.: Elastic materials with two strerss-free configurations. J. Elast. 87, 61-69 (2002)

2. Appell, P.: Figures d'èquilibre d'une mass liquide homogéne en rotation, Paris (1932)

3. Padula, M.: Free work identity and nonlinear instability in fluids with free boundaries. In: Chen, C.C., Chipot, M., Lin, C.S. (eds.) Recent Advances in Elliptic and Parabolic Problems. Proceedings of the International conference, Hsinchu, Taiwan University, 16-20 February 2004, pp. 203-214. World Scientific Publishing, Singapore (2005)

4. Padula, M.: Free work and control of equilibrium configurations. Ann. Univ. Ferrara, Sez.VII (N.S.) 49, 375-396 (2003)

5. Padula, M.: Free work and control of equilibrium configurations.In: Rodrigues, J.F., Seregin, G., Urbano, J.M. (eds.) Trends in Partial Differential Equations on Mathematical Physics, Obidos, 7-10 June 2003. Progress in nonlinear differential equations and their applications, vol. 61, pp. 213-223. Birkhäuser, Basel (2005)

6. Padula, M., Solonnikov, V.A.: Existence of non-steady flows of an incompressible viscous drop of fluid in a frame rotating with finite angular velocity. Elliptic and Parabolic Problems. In: Proceedings of IV European Conference, pp. 180-203. World Scientific Publishing, Singapore (2002)

7. Padula, M., Solonnikov, V.A.: On the stability of equilibrium figures of uniformly rotating liquid drops in $n$-dimensional space. In: Proceedings of the Conference in Kyoto, 6-10 January 2006 (2006)

8. Solonnikov, V.A.: On the stability of axisymmetric equilibrium figures of rotating viscous incompressible liquid. Algebra Anal. 16(2) (2004)

9. Solonnikov, V.A.: On the stability of nonsymmetric equilibrium figures of rotating viscous incompressible liquid. Interfaces Free Bound 6, 461-492 (2004)

10. Solonnikov, V.A.: On instability of axially symmetric equilibrium figures of rotating viscous incompressible liquid. Zap. Nauchn. Sem. POMI 318, 277-297 (2004)

11. Solonnikov, V.A.: On instability of equilibrium figures of rotating viscous incompressible liquid. J. Math. Sci. 128, 3241-3262 (2005)

12. Solonnikov, V.A.: Letter to the editor. J. Math. Sci. 135(6), 3522-3528 (2006)

13. Vladimirov, V.A., Rumiantsev, V.V.: Inversion of Lagrange's theorem for a rigid body with a cavity containing a viscous liquid. Prikl. Matem. Mekhan. 54, 190-200 (1990) 